on the history of, and the prospects and botanical options for, range improvement and rehabilitation. Several tables here include important general information on species characteristics relevant to range improvement.

A 25-page section describing the nature reserves of Uzbekistan is the next major section. Included here are interesting descriptions and photographs of reserves located in the different ecosystems of Uzbekistan.

In the book's final main section, a one-page statement of general conclusions precedes 10 pages of color maps, the book's extensive bibliography, an interesting glossary in English and Cyrillic, appendices of meteorological stations, climatological figures, and phylogenetics of major rangeland plants, a general index, and bios of the authors and acknowledgements of their cooperating organizations.

Any limitations of Rangelands of the Arid and Semi-arid Zones of Uzbekistan likely reflect more the limited information available on the region than limitations of the authors' efforts. In any case, the book's outstanding features overwhelm any limitations in available regional data that might be manifested in the book. An exhaustive summary of admirable features would include diverse technical elements of content and format, but more philosophically, the book exemplifies at least 3 important elements.

First, the book shows how far range management science has come internationally, not just as an application of ecology, but also as a management science integrally involved with a broader culture. Its excellent photographs are particularly effective in binding together the diverse elements of the book's content into a coherent cultural landscape, and should remind Westerners (of a couple of kinds) of the diversity of potential environmental, ecological, and cultural content of range management science. Rarely has range management science seemed more coherent.

Second, plenty of information useful in management is included in a thoroughly bioclimatological, ecological, and cultural context, with a refreshingly natural, unapologetic tone characteristic of a part of the world where grazing on a range is not a discouraging word. Grazing seems a natural part of ecology in this book-more natural that it ever does in current American books on ecology or grazing management, or in books from any region where grazing is not naturally accepted as the major element of the prevailing culture. Rarely has range management science seemed more comfortable.

Third, as a result of (1) and (2), the range management science in the book does not seem economically marginalized to irrelevance by pressures of industrialized, specialized agribusiness and eco-business. Range management science is inherently important for the landscape and the culture. Rarely has range management science seemed more relevant.

Rangelands of the Arid and Semi-Arid Zones of Uzbekistan will be an indispensable reference for range management scientists and range managers in Central Asia, and parts of it will be fascinating reading for individuals involved with rangeland plants, the bioclimatology of steppe regions, and pastoral societies. It will certainly be a valuable referential base for future regional research. In fact, any individual traveling in the drier parts of Uzbekistan who has more than a superficial interest in the climatology and ecology of the region would find this book extremely valuable as a field guide to plants, vegetation types, and climate.

Readers will find photographs of Dr. Gintzburger and his colleagues accompanying their brief bios near the end of Rangelands of the Arid and Semi-Arid Zones of Uzbekistan. Their book is a tribute to how attractively range management science can be packaged and presented to both professionals and to the public. A text that keeps moving, insightful photography, efficient, attractive figures, and concise tables are combined with an energizing format to create an aesthetic scientific contribution. Rarely has range management science ever looked better. - David $L$. Scarnecchia, Washington State University, Pullman, Washington.

\section{Battling Resistance to Antibiotics and Pesticides: An} Economic Approach. Edited by Ramanan Laxminarayan. 2003. Resources for the Future Press, Washington, DC. 377p. US $\$ 65.00$ hardback. ISBN 1-891853-51-1.

Researchers in disparate fields now agree that the growing resistance of bacteria to antibiotics and pests to pesticides threatens to undo some of the most noteworthy advances that have taken place in agriculture and in public health. As a result of this agreement, economists, biologists, and researchers in public health have begun to focus increasing research attention on some of the most vexing questions concerning the phenomenon of resistance. This important edited book consists of 12 chapters and related commentaries. The individual chapters were presented as papers at a conference on the economics of resistance organized by Resources for the Future in April 2001. Rather than proceed with a tedious chapter by chapter account, in the rest of this review, I shall focus on 7 of the 12 chapters. This should give the reader a flavor for the intellectual contributions of this book.

The first 3 chapters analyze issues concerning the optimal management of resistance. Chapter 1 generalizes a previous economic-epidemiological model of the optimal use of antibiotics by allowing for the possibility that there are fitness costs associated with genes that permit a disease to be resistant to treatment with antibiotics. What is the difference between economic and epidemiological control policies? The authors use an optimal control-theoretic framework to shed light on this basic question. The analysis in this chapter tells us that "the economic policy takes into account both the costs today to treat and the increasing costs associated with future treatment because continuing to treat at high levels builds up resistance" (p. 79).

Chapter 2 is a generalization of Chapter 1 in the sense that this chapter studies the case of 2 antibiotics and 2 types of infection. Once again, an optimal control-theoretic framework is used to shed light on the antibiotic treatment regime when resistance is renewable. Although the authors are able to obtain definitive answers only for special cases, they intelligently discuss why it is not possible to determine the optimal solution. As they helpfully point out, their "Hamiltonian is not concave in the state variables, and [hence] the standard sufficiency theorems of Mangasarian or Arrow and Kurz do not apply" (p. 51).

The brief Chapter 3 focuses on the value of treatment heterogeneity for infectious diseases. The authors begin by correctly noting that for well known reasons, treatment homogeneity is valued in the medical profession. This notwithstanding, the authors use a static framework to argue that "from a societal perspective, it may even be desirable to treat some patients with more expensive drugs even while it is individually suboptimal to do so" (p. 67). Put somewhat differently, the basic argument here is that there are circumstances in which it is more desirable to treat an infectious disease with a mixed strategy that calls for the use of an antibiotic cocktail.

Even though the problem of resistance management is fundamentally stochastic in nature, a drawback of the chapters I have 
commented on thus far is that they analyze the problem in deterministic settings. Further, Chapters 1 and 2 study dynamic optimization problems that are linear in the control variables. The structure of the optimal solution for this class of problems is well known and in this sense the analysis in these 2 chapters is a little limiting. Finally, neither Chapter 2 nor Chapter 3 consider the practical difficulties of implementing their "treat with different antibiotics" policies. These lacunae notwithstanding, I would like to note that Chapters 1 through 3 are all noteworthy contributions to a small but growing literature on the management of resistance.

The ways in which stochastic modeling can increase our understanding of the impacts of transgenic crops are amply demonstrated by the competent analyses in Chapters 8 and 9. These chapters present the following 3 useful results. First, the critical value that must arise before a transgenic crop is released is amplified by the presence of uncertainty. Second, in some situations involving $\mathrm{Bt}$ corn, "mandatory refuge areas and tax incentives that might be expected to delay release actually promote early release" (p. 240). Finally, although elementary cost-benefit analysis can be used to justify the release of a transgenic crop, this justification vanishes when one conducts the same analysis making allowance for the presence of uncertainty. Although the modeling in these 2 chapters is versatile, as noted by the commentator, both chapters would have profited from a more detailed discussion of the invasion and persistence of pest resistance.

Chapters 11 and 12 focus extensively on the behavior of firms in markets characterized by the presence of agricultural pest resistance. Specifically, Chapter 11 uses an interesting dynamic economic model to study the tension between what the authors call creative and adaptive destruction. As the authors perceptively point out, although a patent based incentive mechanism does pro- vide the capacity to capture rents from an innovation, this mechanism "also provides the prospect that the stream of rents may be truncated because of a competitor's innovation" (p. 299). In addition, it is salient to comprehend that the stream of rents from an innovation may be curtailed because of biological forces. The interaction of these 2 factors means that a decentralized biotechnology sector will not pursue the objectives that a social planner would like it to pursue.

The terminal Chapter 12 makes one salient point and that point is this. It is not necessarily the case that "resistance provides incentive to overapply pesticides..." (p. 350). In particular, because pesticide manufacturers are likely to have a monopoly on the production of pesticides during the life of a patent, it is possible that farmers will actually underapply pesticides relative to the optimal solution. This is an engaging chapter and it does contain an expansive discussion of the economic, institutional, and scientific factors that influence the use of pesticides and the subsequent evolution of pest resistance. Even so, this chapter would have profited from a more rigorous and analytical discussion of the above mentioned factors that it so helpfully identifies.

Let me conclude this review by noting that this book is both important and interesting. It is one of those rare edited books in which virtually every chapter is of high quality. In addition, the book contains perspicacious commentaries by scholars on the individual chapters and these commentaries themselves are a pleasure to read. Consequently, I enthusiastically recommend this book to all readers who wish to learn more about the ways in which modern interdisciplinary analyses can shed light on the problems associated with the increasing resistance of bacteria to antibiotics and pests to pesticides.-Amitrajeet A. Batabyal, Rochester Institute of Technology, Rochester, New York.

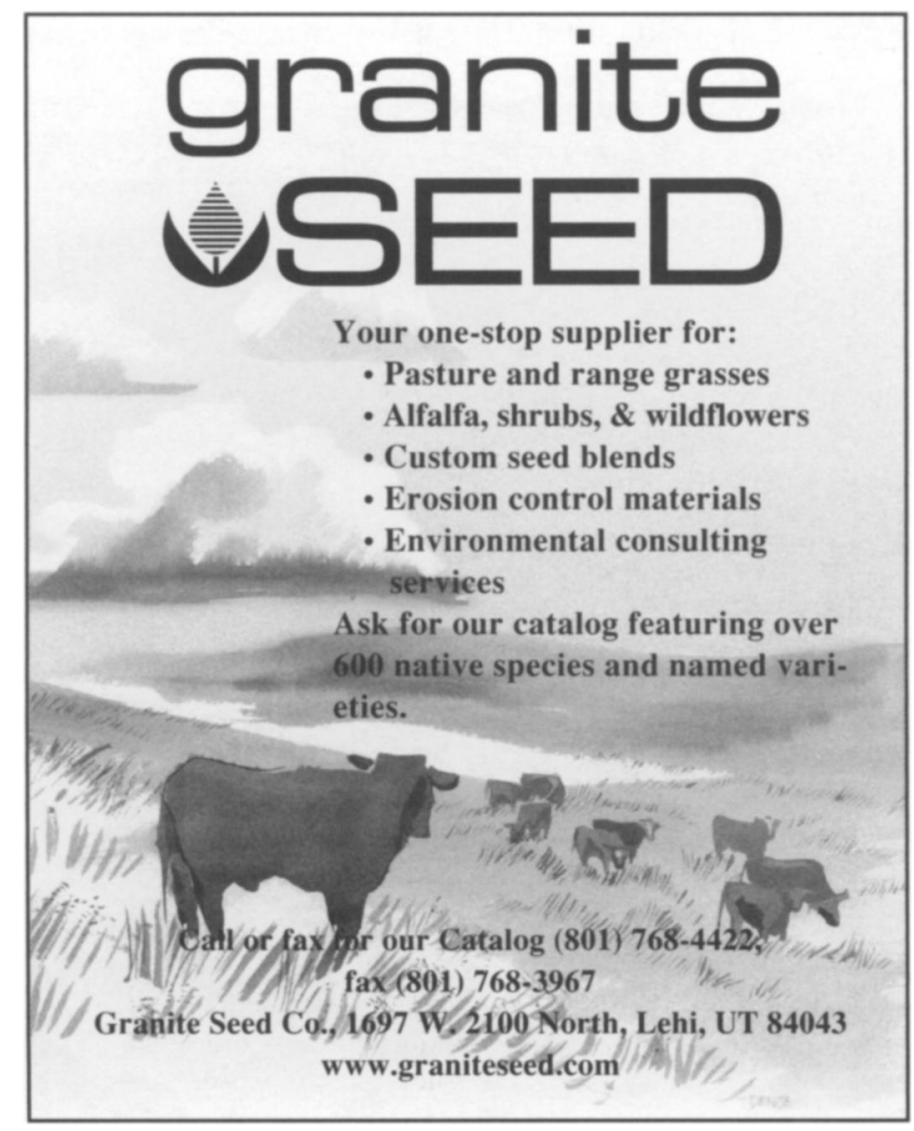

